\title{
Anatomy is the great ocean of intelligence upon which the true surgeon must sail. chylous cyst: Presenting as supraclavicular mass
}

\author{
Shalina Ray ${ }^{1}$, Kavitha P Palled ${ }^{2, *}$, Anitha Kumari ${ }^{3}$, Divya ${ }^{4}$ \\ ${ }^{1,3}$ Consultant, ${ }^{2}$ Senior Registrar, ${ }^{4}$ DNB Resident, ${ }^{1-4}$ Dept. of ENT, ${ }^{1-4}$ Manipal Hospital old Airport Road Bangalore, Karnataka, India \\ *Corresponding Author: Kavitha P Palled \\ Email: bkkavita001@gmail.com
}

\begin{abstract}
Cysts of the thoracic duct located in the supraclavicular region are uncommon. To date only 12 cases in this topographic area have been described in the literature. Lymphocoele of the thoracic duct, alternatively referred to as a thoracic duct cyst, is an uncommon abnormality that can present rarely as a left neck mass arising from the supraclavicular fossa. Recognition of the aetiology of such a neck mass on imaging is crucial to avert unnecessary intervention and, where surgical intervention is planned, to avoid both unexpected findings at surgical exploration and secondary complications. We present such a case of a cervical neck mass. Thoracic duct cyst are relatively unclear etiology. B The first reported case of thoracic duct cyst was in 1964 by steinbergetal. Since that time, only 32 cases have been reported in the literature with the largest case series consisting of only 5 patients. 2 The most common approach to managing these lesions is surgical excision with ligation of the thoracic duct. We report a case of thoracic duct cyst involving the left supraclavicular fossa.
\end{abstract}

Keyword: Cyst.

\section{Introduction}

Cysts of the thoracic duct located in the supraclavicular region are uncommon. To date only few cases in this topographic area have been described in the literature. We now report this extremely rare case of cervical thoracic duct cyst in the left supraclavicular fossa. Recognition of the aetiology of such a neck mass on imaging is crucial to avert unnecessary intervention and, where surgical intervention is planned, to avoid both unexpected findings at surgical exploration and secondary complications. Emerson (1950) described the first case diagnosed at surgery during the patient's lifetime. He found four previous reports of supradiaphragmatic

Thoracic duct cysts diagnosed post-mortem, the first by Enzmann in 1883. ${ }^{1,2}$ Tsuchiya et al (1980) reviewed eight cases described since 1950 and added one case which they believed to be the first diagnosed before thoracotomy. ${ }^{2}$

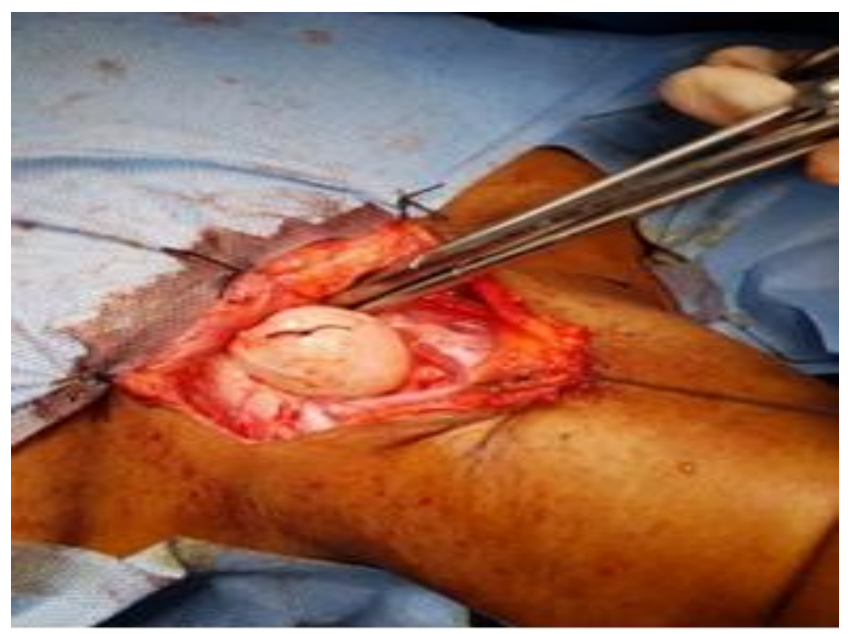

Fig. 1: Intra-Operatively showing cystic mass occupying the left supraclavicular region

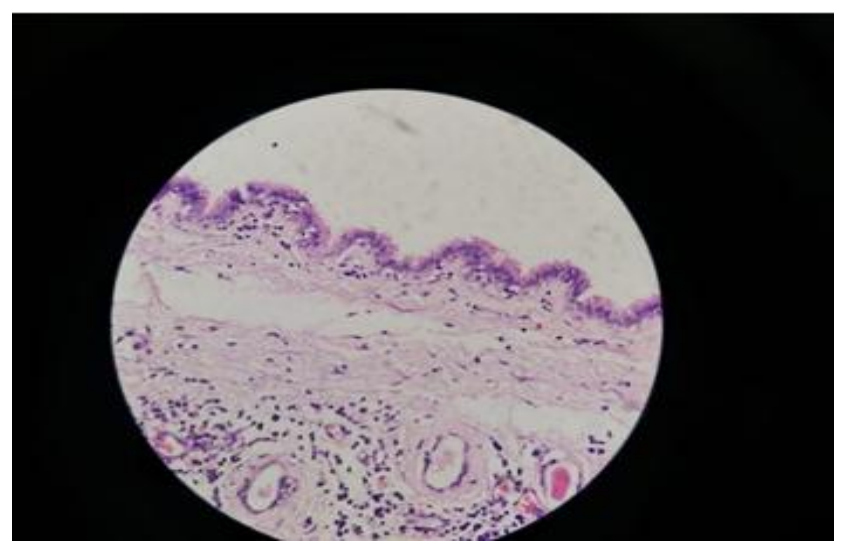

Fig. 2: Histopathological findings showed that cyst wall contained an inner single layer of flat endothelial cells, smooth muscle cells and islets of lymphoreticular tissue.

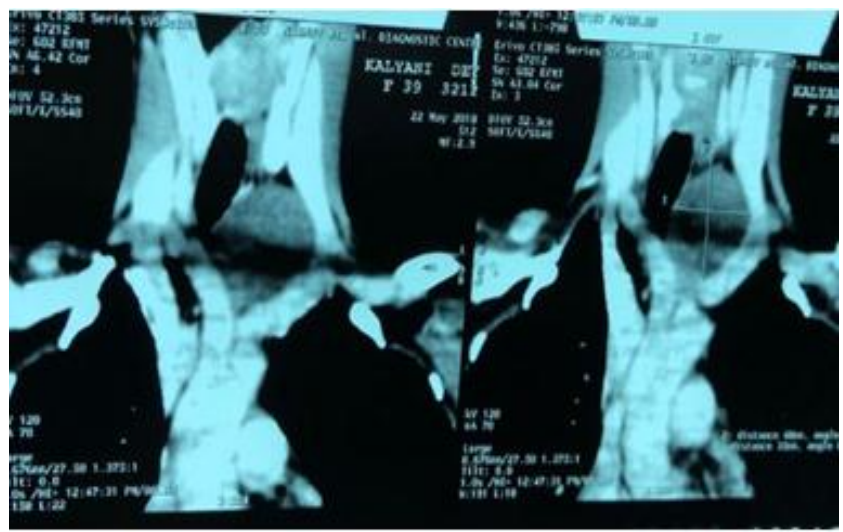

Fig. 3: On computed tomography with contrast showed a thin walled, non-enhancing monocystic mass pushing the trachea, related to carotid sheath. 


\section{Case Report}

A 38 year old female presented to our ear, nose and throat clinic with a 4 month history of a painless, nonpulsatile lump in the left supraclavicular fossa. She had noticed it only incidentally and did not feel that it had increased in size over the 4 months. There was no history of any subjective neurological abnormality of the left upper limb. The patient was otherwise fit and well, was a nonsmoker and consumed alcohol only very occasionally. There was no history of notable trauma in the past and no history of surgery. Examination identified a cystic, non-tender mass, compressible swelling, in the medial left supraclavicular fossa. On computed tomography with contrast showed a thin walled, non-enhancing monocystic mass pushing the trachea, related to carotid sheath. FNAC, aspirated fluid yellowish milky in appearance, reported mass almost disappeared after aspiration, but reappeared. Surgical excision of the cyst with ligation of the lymphatic duct branch performed. Post-operative period was unremarkable. Histopathological findings showed that cyst wall contained an inner single layer of flat endothelial cells, smooth muscle cells and islets of Lymphoreticular tissue.

\section{Discussion}

Lymph is delivered to the venous circulation via the right and left cervical lymphovenous portals, which are located at or near the junctions of the internal jugular and subclavian veins where these veins converge to form the right and left brachiocephalic veins. On the right, three main lymph trunks, the (right) jugular trunk, the (right) subclavian trunk and the (right) bronchomediastinal trunk, converge at their venous junction. On the left, there are three similarly named trunks plus a fourth trunk, the largest, called the thoracic duct. ${ }^{3,4}$

Well-known cysts in the cervical region include median cervical cyst, lateral cervical cyst and Cystic lymphangioma. However, thoracic duct cyst is very rare.

Enzmann (1883) quoted by Emerson (1950) described a post-mortem finding similar to the present case. Near the upper end of the thoracic duct an egg-shaped, smooth cyst was found, with a clot plugging the entrance into the left subclavian vein. This cyst was assumed to be caused by mediastinitis ${ }^{2,3}$ and. ${ }^{4}$ Barlow and Gracey (1965) described a 76-year-old woman with a gradually enlarging swelling in the left side of the neck of 2 years' duration, Surgery disclosed cystic dilatation of the thoracic duct containing clear fluid and communicating with the afferent thoracic duct $^{1}$.

Ross (1961) recognised two varieties of duct cysts: the lymphangiomatous (congenital) and the degenerative (acquired). As obstruction by ligation will not cause a cystic dilatation, a combination of degenerative change and obstruction may be postulated as a cause for the nonlymphangiomatous acquired cysts., 5 Numerous theories have been suggested to explain the aetiology of lymphocoele of the terminal thoracic duct. Both congenital weakness in the thoracic duct wall and acquired degenerative process from inflammation have been proposed as causative mechanisms. Trauma has also been suggested as the underlying aetiology. ${ }^{6,7}$

The differentiation of thoracic duct cyst from cystic lymphangioma is most important. In general, cystic lymphangioma is congenital and frequently observed in infants, developing by two years of age in $90 \%$ of the cases. The cyst is multilocular and lined by smooth epithelium and contains serous or yellow transparent fluid. Awareness of the anatomical course of the thoracic duct and of the existence of morphological anomaly is therefore paramount. ${ }^{4,8}$

The diagnosis of cervical thoracic duct is suspected by a complete physical examination and radiologic studies. Physical examination demonstrates a left supraclavicular fluctuant and painless mass, with possible intermittent swelling. Ultrasound-guided FNA can be extremely helpful. Lymph (or chyle) has a very distinct appearance on gross inspection, exhibiting a milky appearance. It also has a very unique composition and so aspirate should be sent for both cytological and biochemical analysis to consolidate the diagnosis. ${ }^{8,9}$ Lymphomatous lymph nodes in the neck can demonstrate a pseudocystic appearance on ultrasound assessment, but their underlying lymphoproliferative process is revealed on CT or MRI. CT scan demonstrates the cystic nature of the lesion and specifies anatomic connections of the cyst with the thoracic duct and other vessels. Specificity is increased by addition of an orally ingested mixture of ethiozed oil, which enhances visualization of the thoracic duct ${ }^{7,8}$, and. ${ }^{9}$ Lymphography with lipiodol injected peripherally, had been also advocated to demonstrate thoracic duct cysts of the mediastinum.

The general management of asymptomatic thoracic duct cyst is observation of its progress. There are no reports of malignant changes in asymptomatic cases. Surgery is indicated in patients with clinical symptoms such as pain or compression of the surrounding tissue. The surgical removal is performed by a limited cervical incision along the anterior border of the stercleidomastoid muscle, with ligation of the duct at the bottom and the top of the cyst. Repeated cyst puncture and infusion of a sclerotic agent into the cyst may be undertaken. However, the risk of infection increases in the former, and there are no precedent cases in the latter. Further evaluation of this method is needed. ${ }^{4,6,8}$

\section{Conclusion}

Chylous cyst are very rare entities which may present with a wide variety of symptoms. We report a left supraclavicular mass, thoracic duct cyst must be taken into consideration. Knowledge of the normal course of the thoracic duct, particularly in relation to its cervical course, and of the morphological variations that it can demonstrate, including the rare formation of a lymphocoele, is invaluable in distinguishing morphological anomalies from pathological lesions of the lower cervical region therefore radiological findings and appearance of the aspirated fluid from the cyst is helpful for the diagnosis. We advocated surgical treatment of the cyst in order to confirm the diagnosis by histological analysis and to prevent potential 
complications as spontaneous or traumatic rupture of the cyst.

\section{Conflict of Interest: None.}

\section{References}

1. Barlow D, Gracey L. Cystic dilatation of the thoracic duct. $\mathrm{Br}$ J Clin Pract 1965;19:101-102.

2. Kolbenstvedt A, Aanesen J. Cystic dilatation of the thoracic duct presenting as a supraclavicular mass. Br J Radiol 1986;59:1228-1229.

3. Wax MK, Treloar ME. Thoracic duct cyst: an unusual supraclavicular mass. Head Neck 1992;14:502-505.

4. Sinclair D, Woods E, Saibil EA, Taylor GA. Chyloma: a persistent post-traumatic collection in the left supraclavicular region. J Trauma 1987; 27:256-259.

5. Livermore GH, Kryzer TC, Patow CA. Aneurysm of the thoracic duct presenting as an asymptomatic left supraclavicular neck mass. Otolaryngol Head Neck Surg 1993;109:530-533.

6. Mosabehi A, Gleeson M, Owen WJ. Mass in the neck after Wishplash injury. J R Soc Med 1998;91:493-494.

7. Seelig MH, Klinger PJ, Oldenburg WA. Treatment of a postoperative cervical chylous lymphocele by percutaneous sclerosing with povidone-iodine. J Vasc Surg 1998;27:11481151.

8. Tsuchiya R, Suguria Y, Ogata T, Suemasu K. Thoracic duct cyst of the mediastinum. J Thorac Surg 1980;79:856-859.

9. Emerson GL. Supradiaphragmatic thoracic duct cyst. Engl J Med 1950;242:575-578.

How to cite this article: Ray S, Palled KP, Kumari A, Divya. Anatomy is the great ocean of intelligence upon which the true surgeon must sail. chylous cyst: Presenting as supraclavicular mass. Indian J Anat Surg Head Neck Brain 2018;5(1):34-36. 